Itinéraires Itinéraires

Littérature, textes, cultures

2010-2 | 2010

Les blogs

\title{
L'exercice autoritatif du blogueur et le genre éditorial du microblogging de Tumblr
}

Évelyne Broudoux

\section{(2) OpenEdition}

Journals

Édition électronique

URL : http://journals.openedition.org/itineraires/1941

DOI : 10.4000/itineraires. 1941

ISSN : 2427-920X

Éditeur

Pléiade

Édition imprimée

Date de publication : 1 juillet 2010

Pagination : 33-42

ISBN : 978-2-296-12012-9

ISSN : 2100-1340

Référence électronique

Évelyne Broudoux, "L'exercice autoritatif du blogueur et le genre éditorial du microblogging de Tumblr», Itinéraires [En ligne], 2010-2 | 2010, mis en ligne le 01 juillet 2010, consulté le 02 mai 2019. URL : http:// journals.openedition.org/itineraires/1941 ; DOI : 10.4000/itineraires.1941

\section{(c) $\oplus \Theta \Theta$}

Itinéraires est mis à disposition selon les termes de la licence Creative Commons Attribution - Pas d'Utilisation Commerciale - Pas de Modification 4.0 International. 


\title{
L'exercice autoritatif du blogueur et le genre éditorial du microblogging de Tumblr
}

\begin{abstract}
This article as an intermediary stage in a research - on the relations of « publication », "social networks" and "web of documents" concepts - is questioning the conditioning of the publishing genre by online communication tools. Four months of participant observation with the help of a microblogging tool (Tumblr) in 2008 show modifications in style and authors' behaviors.
\end{abstract}

Keywords : publishing genre, authorship, monograph, microblogging, list Mots clés : genre éditorial, auctorialité, monographie, microblogging, liste

La dualité autoritative est rendue visible sur un certain nombre de blogs issus d'horizons variés (personnels, politiques, littéraires, scientifiques, etc.). Une oscillation se laisse effectivement observer entre le « placement de soi » dans un espace social et un domaine d'activités et la " production d'un contenu original » attribuable à un auteur. En effet, ces blogs se laissent reconnaître à leurs prises de position, aux interpellations diverses qui rythment les contributions. Et il est quelquefois difficile de distinguer ce qui au fond motive leur auteur : être reconnu dans un champ pour ses qualités personnelles ou bien produire et créer des objets dont la valeur sera appréciée par un public constitué de néophytes ou d'experts. Doit-on prendre ces manifestations pour des affirmations d'auteurs en manque de légitimité ou s'agit-il « simplement » d'une construction auctoriale classique, à partir de laquelle l'auteur est en mesure de laisser s'exprimer l'écrivain?

Cependant, l'exercice autoritatif du blogueur se déroule dans un univers techniquement contraint et l'observation de l'empreinte de l'outil permet aussi bien de constater des prescriptions et des inductions dans les contenus produits que dans les comportements. 


\section{Genre éditorial et blogging}

Alors que le genre littéraire survit aux changements de supports en s'adaptant aux nouvelles formes de communication, le genre éditorial est par définition encadré par l'éditeur qui sélectionne et agence les contenus selon les supports. Sa constitution est donc directement dépendante de la matière qui accueille les produits de l'écriture ${ }^{1}$. Par exemple, dans la presse imprimée, le genre du reportage, du portrait, de la brève, et même du roman-photo sont clairement identifiés. Ainsi la brève, information par définition courte, se logera selon la largeur de la page, dans des colonnes étroites encadrant un article plus long. Cela nous permet d'affirmer que les formes matérielles de production et de diffusion des contenus interfèrent avec leur traitement stylistique. Citons, par exemple, le « quarante-cinq tours », ancienne petite galette de vinyle à deux faces, qui est à la fois support et genre éditorial, puisque les morceaux limités en durée y étaient sélectionnés en fonction de leur capacité à récolter du succès. Récemment, on a pu observer des interrogations quant à la survie du genre « album » musical, où l'enchaînement des morceaux participe à la création du tout. Dans un autre domaine, celui des publications scientifiques en ligne, l'article est consulté prioritairement avant la revue qui l'a accueilli. Avec la numérisation, on observe donc des usages se déplaçant vers la consultation ou l'achat à l'unité, plutôt que sur les formes éditoriales classiques.

Depuis bientôt une dizaine d'années, une famille d'outils-logiciels ${ }^{2}$ a fait son apparition, destinée à faciliter la publication sur le Web. Sa caractéristique est d'intégrer les différentes phases éditoriales du processus de sélection des contenus dans un workflow automatisé. Un texte, une image peuvent être enregistrés en tant que drafts $^{3}$ dans le système par un rédacteur, qui pourra le reprendre plus tard et le publier directement avec ou sans validation suivant le paramétrage du système choisi. Les plates-formes de blogs sont typiquement des CMS. Aussi, le genre du blog est-il éditorial ${ }^{4}$ car ce sont les gabarits du système de publication qui imposent leur débit et leur forme à ce qui est publié.

Dans la courte histoire des outils d'auto-publication, la famille des blogs s'est récemment diversifiée. D'abord nommée Tumblelog ${ }^{5}$, une variante a

1. Ainsi, Walter Benjamin remarque dans «Le narrateur. Réflexions à propos de l'œuvre de Nicolas Leskov» (Mercure de France, t. 315, n 1067, mai-août 1952) que le genre du roman est directement dépendant du livre à partir duquel il peut se propager.

2. Ce sont les CMS : Content management systems ou Systèmes de gestion de contenus.

3. « Premier jet ».

4. Évelyne Broudoux, «Outils informatiques d'écriture et de lecture : nouvelles conditions au "devenir auteur" ». Communication présentée à Écritures en ligne : pratiques et communautés, colloque Cercor/Sfsic, université Rennes 2, 26 septembre 2002. En ligne, sur ArchiveSic : http://archiveSIC.ccsd.cnrs.fr/documents/archives0/00/00/07/96/index_fr.html, consulté le 7 avril 2010.

5. Selon Wikipedia, le terme est issu de l'anglais tumble : pêle-mêle et $\log$ : journal de bord. 
favorisé l'édition de notes prises à la volée utilisée pour partager rapidement des idées, des découvertes, des créations. Pour ce faire, les systèmes proposent des billets spécialisés selon leur contenu (textes, liens, images, vidéos, etc.). Le système Tumblr fait partie de cette première génération d'outils à spécialiser les billets selon la matière qu'ils réceptionnent. Un billet Tumblr est préformaté selon sa forme sémiotique : simple lien hypertexte, texte ou image, enregistrement sonore ou vidéo, ou symbolique : citation avec sa source ou republication entière d'un « post».

Le microblogging, qui semble être l'expression du moment englobant la notion de Tumblelog, est un raffinement du genre. Produits directement sur le Web ou sur mobile, les billets de plus en plus courts deviennent de simples messages textuels, comme pour Twitter où leur taille limite n'excède pas 140 signes. Leur forme est alors plus proche de la messagerie instantanée et l'outil de publication se transforme en outil de gestion de conversations.

La particularité de ces micropublications est qu'elles se déroulent devant un double public : celui des internautes et celui plus restreint des « abonnés » formant le réseau social du blogueur. Le microblogueur a alors accès à l'ensemble des billets publiés par son réseau dans une interface publique ou privée selon les choix proposés par le système. L'abonnement à un blog n'étant pas réciproque, de multiples groupes coexistent dans des communautés d'intérêts fragmentées qui se bâtissent selon des recommandations manuelles ou automatisées.

Rappelons que Carey avait mis en évidence dans ses études les effets structuraux des technologies de communication, en repérant les effets de décontextualisation du télégraphe et son influence sur la rédaction des contenus des journaux au XIX ${ }^{\mathrm{e}}$ siècle aux États-Unis ${ }^{6}$. On est ainsi passé d'une nouvelle locale concernant une communauté de lecteurs circonscrite par le lieu, à une information décontextualisée susceptible d'être lue par un public façonné d'habitudes et de références non partagées. L'information a remplacé la nouvelle et devait pouvoir être lue à plusieurs centaines de kilomètres et être comprise. Son style en a immédiatement été affecté : moins d'ironie et de journalisme de révérence réservés aux happy few. De plus, la technologie qui supposait une écriture faite de précision et de rapidité sépara l'observateur du scripteur. Sauf exception comme l'éditorial ou le billet, le rédacteur ne devait plus s'investir personnellement dans l'information qui devait rester la plus "objective » possible, rapprochant en cela la prose journalistique des formes du discours scientifique.

Si nous admettons qu'une technologie d'inscription et ses réseaux spécifiques de diffusion ou d'acheminement sont susceptibles de modifier le genre du contenu rédactionnel, des manifestations récurrentes caractérisant le blogging sont repérables.

6. James W. Carey, Communication as culture. Essays on media and society [1988], New York, Routledge, 1992. 


\section{La spécification des voix}

Le modèle éditorial du blog a pour particularité de spécifier les voix par une séparation nette entre auteur du blog et commentateurs et l'automatisation de leur signature. Il place le blog au sein d'un tissu social (lecteurs et blogosphère) grâce aux rubriques constituées de liens hypertextes (blogroll, permalink, trackback, fil $\mathrm{RSS}^{7}$ ).

S'il existe des blogs collectifs $\left(\right.$ AEIOU $\left.^{8}\right)$ pouvant même se transformer en revue (Homo numericus $^{9}$ ), la majorité des blogs est tenue de manière individuelle. Et les plates-formes de blogs de type Tumblr sont bâties sur ce principe (un blog = une personne), même si à partir d'un compte personnel, il est possible d'ajouter un blog prévu pour être alimenté à plusieurs. De nombreuses marques stylistiques typiques de l'investissement personnel du blogueur sont alors repérables et indiquent un discours subjectivé. Le lecteur peut être assimilé à un " proche », il est alors apostrophé, pris à témoin, la relation fantasmée auteur-lecteur est mise en scène par le blogueur. Les formes verbales conversationnelles sont alors dominantes (ex. : impératif) appuyées par les formes directes du tutoiement ou du vouvoiement et l'emploi de la première personne du singulier. Cela donne l'impression que l'auteur du blog s'adresse à un cercle restreint de lecteurs sur un ton confidentiel suggérant que « ceux à qui je m'adresse me comprendront » (voir fig. 1, 2, 3).

Par la multiplicité des voix susceptibles de réagir et de laisser des traces lisibles, l'information continue de se transformer après publication : jeu de citations, pluralité des commentaires, re-blogs, retours en arrière et corrections, le processus de digestion sociale de l'information est rendu visible par les commentaires et les blogs connectés. Ce jeu a pour particularité de transformer la notion de publication en conversation, puisque tout texte est appelé à être immédiatement remanié par les lecteurs, les blogueurs ou même leur auteur initial.

Remarquons aussi le renouveau de la rature. Alors qu'elles sont habituellement utilisées à l'étape de la relecture papier pour améliorer les textes, leur fonction change lorsqu'elles sont intégrées dans des documents publiés sur le Web :

- soit elles servent à indiquer une correction faite après publication, quelquefois après une remarque de lecteur, comme le montre cette offre d'emploi [fig. 1];

7. Évelyne Broudoux, Sylvie Grésillaud, Hervé Le Crosnier et Véronique Lux-Pogodalla, "Construction de l'auteur autour de ses modes d'écriture et de publication », dans Imad Saleh et Jean Clément, Créer, jouer, échanger : expériences de réseaux, H2PTM'05, Paris, Hermès science, 2005, p. 123-142. En ligne, sur ArchiveSic : http://archivesic.ccsd.cnrs. fr/sic_00001552, consulté le 7 avril 2010.

8. http://www.fluctuat.net/blog/.

9. http://www.homo-numericus.net/. 
- ou bien, par leur typographie, elles renforcent une opinion, un remords, une contradiction, survenus dans la tête du blogueur au moment de la rédaction du billet [fig. 2 et 3].

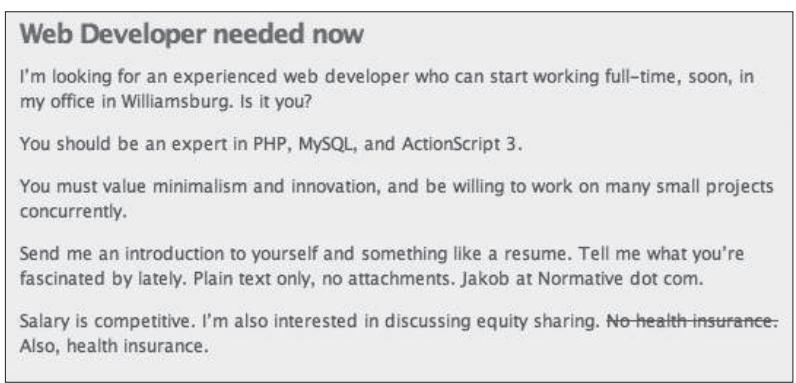

Fig. $1^{10}$

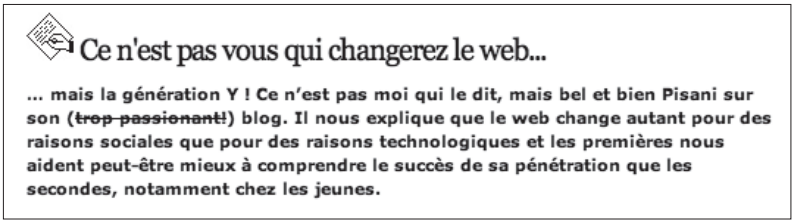

Fig. 2

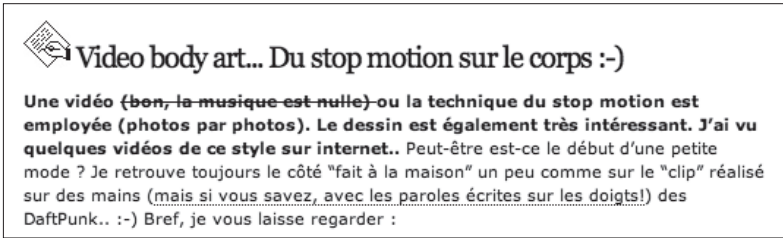

Fig. 3

Le passage du blogging au microblogging transforme le concept du blog en tant qu'espace suscitant un dialogue entre auteur et lecteurs, car l'espace réservé aux retours du public disparaît ou devient optionnel. Dans Tumblr, le blogueur « re-blogue » un billet d'un autre blogueur lorsqu'il veut le partager avec son réseau, Twitter est en passe d'automatiser le même service par des « re-tweet », et il est possible de désactiver la fonction « commentaire » sous

10. « Recherche Développeur web de suite/ Je recherche un développeur web expérimenté qui pourrait commencer à travailler à plein-temps, rapidement, dans mon bureau à Williamsburg. Est-ce que c'est toi? Tu dois être un expert en PHP, MySQL, et ActionScript 3./ Les valeurs de minimalisme et d'innovation doivent être les tiennes, et tu dois te tenir prêt à mener de front plusieurs petits projets./ Envoie-moi une présentation de toi et quelque chose comme un CV. Dis-moi ce qui t'a fasciné récemment. Seulement du texte, pas de pièces jointes. Jakob à Normative point com./ Le salaire est compétitif. Je peux aussi discuter des intéressements. Pas de sécurité sociale. Si, en plus, sécurité sociale.» 
Friendfeed. Sur Twitter, le simple lecteur s'efface, l'intérêt étant de participer et de créer son réseau. Les voix sont donc « égales » à première vue. En réalité, la répartition en « suiveurs » et « suivis » fait apparaître des zones d'influence et crée des têtes de ponts qui vont rapidement se démarquer des autres.

\section{Les prescriptions de l'outil et le genre induit}

Chaque système de gestion de contenu que constitue un blog formate un ensemble de possibilités d'écriture, d'illustrations, de mise en scène, de diffusion. On peut parler de prescription par l'outil puisque ce sont ses fonctionnalités qui vont influencer des styles de publication et des comportements.

Tout se passe comme si le copiage de fonctionnalités d'un outil à l'autre et l'élimination d'options créaient une autre famille d'outils et prescrivait un ensemble de conduites. Les « billets » ultracourts ne reflètent que l'activité du moment ou bien signalent des événements, des liens à visiter. Place à la connectivité et à l'immédiateté : SMS, billets et mails sont publiés depuis les téléphones mobiles. Tout semble indiquer une course sans fin pour rester dans un présent éternel.

Il est intéressant de remarquer ici que l'adoption de Twitter par les blogueurs a modifié leur travail. Plus précisément, alors que cette famille logicielle favorise une prose de l'instantané, les anciens blogueurs ayant conservé leur système de publication ont apporté un soin plus grand à la rédaction de leurs billets qui sont devenus plus rares mais aussi plus longs, rassemblant davantage des synthèses réfléchies que des réactions épidermiques.

De leur côté, les lecteurs de la blogosphère qui se composaient de simples internautes - et aussi bien sûr, de nombreux veilleurs : chercheurs, journalistes, etc. - se sont donc vus sommés de participer pour « rester dans la conversation » mais la sélection n'est-elle pas plus grande encore pour ceux qui n'ont pas pris l'habitude de fréquenter ces nouveaux agencements communicationnels? Ainsi, il serait illusoire de croire que les nombreux réseaux d'intérêts créés convergent vers une agora partagée où chacun serait tour à tour suivi et suivant. Non seulement cette agora n'existe pas, mais ces systèmes fragmentent les communautés d'intérêts en une multiplicité de petits groupes rassemblés autour de personnes phares. C'est ainsi que, sur Tumblr, il est courant de voir une image rebloguée un temps disparaître puis réapparaître plusieurs semaines ou mois plus tard dans un autre sousgroupe. Si l'on imagine que c'est une caractéristique à généraliser aux « réseaux sociaux », il devient difficile de suivre le trajet d'une communauté. En revanche, la capacité d'un groupe à gérer le collectif sera évaluée selon les dispositifs qu'il mettra en place pour parer à cette déperdition d'énergie. Enfin, en l'absence de vue générale et de volonté de prendre du recul sur des événements, survient l'hyperfocalisation qui est l'attention exagérément portée au détail au détriment de la vue d'ensemble. Cette hyperfocalisation se donne à observer sur l'encyclopédie Wikipédia lorsque des « rameaux » 
de connaissance d'un domaine sont développés en l'absence ou l'ignorance systématique des branches maîtresses qui les portent.

\section{Autoréférence et monographies}

Autre phénomène caractéristique du genre induit, les phénomènes d'autoréférence qui émaillent les blogs individuels apparaissent souvent peu maîtrisés par leurs auteurs.

Un des proches collaborateurs de Tumblr, Jakob Lodwick, également musicien, en a ainsi été victime : en se mettant directement en scène par photographies interposées systématiquement prises à des moments " privés » (ex. : bronzage sur la terrasse) ou bien en faisant des montages autoréférentiels d'une interview donnée dans un magazine ou de sa page Facebook, il a donné libre cours à son narcissisme qui a révélé ses faiblesses. Cette surexposition de soi a agacé ses «suiveurs" qui ont entrepris de le punir en publiant des photos peu à son avantage jusqu'à ce qu'il soit poussé à disparaitre du système qu'il avait contribué à créer. Comme si les fans avaient décidé de supprimer l'idole montée sur un piédestal. Disparu pendant plus d'un an de la scène Internet, le blogueur est ensuite revenu sur la plate-forme avec un prénom légèrement modifié, Jake Lodwick, et un projet plus mûr de gestion identitaire. Cela est révélé sur son site par la conjugaison à la troisième personne employée pour se présenter, « Here are things Jake Lodwick likes or made », et un projet personnel de vidéo performance ${ }^{11}$.

C'est évidemment le phénomène d'autopublication qui facilite et amplifie l'autoréférence. En effet, l'éditeur qui est le garant de la qualité des textes est aussi le garant des genres publiés. En dehors du genre autofictionnel, les incartades de la personne écrivante dans le texte d'auteur publié sont rarement admises. Il y a donc éclatement des genres traditionnels.

Ce sont surtout les jeunes intellectuel-le-s qui vont se servir du blog comme d'un espace servant à la construction et à la gestion fine de leur identité : étudiants, jeunes journalistes, artistes ou écrivains, sont susceptibles de jouer avec leur identité, par de multiples détournements passant quelquefois par l'anonymat.

Ainsi, girl.deconstructed signale l'arrêt de son blog qu'elle alimentait depuis deux ans. En cause, une série de billets impubliables qu'elle garde en brouillon, billets qu'elle juge les meilleurs, mais le risque qu'on découvre son

11. Même s'il a fait disparaître les archives de son premier Tumblr, la continuité avec ses anciennes publications est maintenue puisque subsiste sur le Web une page (http://jakoblodwick.com/) indiquant :

- «The name "Jakob Lodwick" has been retired.»

- « Jake Lodwick's tumblr (http://jakelodwick.tumblr.com/). »

- «Odwick.com». 
identité à la lecture de ses archives et que son entourage puisse suivre ses diverses « introspections » la dissuade de continuer. Le 21 mai 2008, elle écrit :

I wrote a deeply personal blog post today. I spent a lot of time on it, reading and rewriting, pushing myself to be as honest as possible. And then I didn't post it.

This one ended up as a private Tumblr post, but it's no different from the 30-plus unpublished drafts idling in my WordPress account. Together, these posts offer a fuller picture of who I really am than all published blog posts combined. It's some of the best stuff I've ever written. I'd love to put it out there for all to read, get feedback (praise! adoration!) and indulge in the serene satisfaction that surely comes with being completely open and honest.

I just can't. Partly because I'm afraid that a coworker (or, even worse, my boss) might stumble upon this here site and read my archives and summarily fire me for the many posts about hating my job and spending full workdays tooling around on the Internet. Partly because I fear the harsh judgment of anonymous commenters. And partly because I fear that even the people who love me the most would be semi appalled (or fully appalled!) by some of the things I'd say if I was being truly confessionnal ${ }^{12}$.

Autre exemple, Agsystems et Firmaerror sont deux facettes d'un même personnage appartenant à Leonel Cunha, peintre portugais, photographe et vidéaste numérique, adepte d'arts génératifs. Ses deux Tumblr ne lui servent pas seulement d'espaces de stockage d'images et de vidéos, mais également de sites miroirs capables de dialoguer, alimentés par deux sources principales distinctes appartenant aussi à l'artiste. Il s'agit donc d'éléments participant à un positionnement d'auteur sur le réseau. L'utilisation de pseudonymes est un fait à remarquer sous Tumblr lorsqu'il est l'occasion pour un même acteur de différencier des activités professionnelles d'activités militantes ou bien de rester en contact avec des proches.

Nous avons vu que la matrice du blog ou du microblog prescrivait des comportements. Le genre induit possède des caractéristiques de contenu

12. «J'ai écrit un billet personnel fouillé aujourd'hui. J'ai passé beaucoup de temps sur celui-ci, à le relire et à le réécrire, en me forçant à rester la plus honnête possible. Et puis je ne l'ai pas publié.

Il a terminé comme un billet Tumblr privé, mais il n'est pas différent des plus de trente billets non publiés restant sur mon compte WordPress. Ensemble, ces billets offrent une image plus complète de ce que je suis vraiment que l'ensemble de tous mes billets publiés. Ce sont les meilleures choses que j'ai jamais écrites. J'aurais aimé les mettre ici à lire pour tous, avoir un retour (acclamation! adoration!) et jouir sereinement de la satisfaction qui arrive sûrement lorsque l'on est entier et honnête.

Je n'ai pas pu le faire. J'ai peur qu'un collègue (ou, pire, mon patron) puisse tomber là-dessus et puisse lire mes archives et me vire illico pour tous ces billets écrits sur ce boulot que je déteste et toutes ces journées entières de travail passées à bricoler sur Internet. En partie aussi parce que j'ai peur du jugement dur des commentateurs anonymes. Et en partie parce que j'ai peur que même les gens qui m'aiment soient à moitié horrifiés (ou totalement horrifiés) par certaines des choses que j'ai dites lorsqu'elles étaient vraiment confidentielles. » 
qui lui sont propres. Pour Tumblr, il s'agirait plutôt de monographies, et les fonctionnalités de l'interface n'y sont pas étrangères car elles encouragent la création de collections thématiques qui se limitent à rassembler des objets sans toutefois les classer ni les évaluer. Au contraire des serveurs de signets ${ }^{13}$ qui réalisent des listes thématiques en collectif, ici, chacun révèle ses marottes de manière individuelle. Les tumbleristes se spécialisent ainsi et se donnent souvent pour objectif de publier régulièrement selon une thématique choisie. Ainsi, lpcoverlover et 365 daysofmusic publient chacun quotidiennement une pochette d'album et une chanson, Buzzle publie plusieurs fois par jour des citations issues de faits de la vie politique française relevés sur divers médias, Fuckyeahgooglesearch rassemble les requêtes les plus populaires ou énigmatiques sur le moteur de recherche de Google. Les collections de Loichay sont constituées par un choix d'infographies illustrant les tendances du Web 2.0, postées sans aucun commentaire; son blog est sous-titré : « Vous voyez ce que je veux dire? » Le graphiste Peekasso publie une sélection de brouillons intermédiaires à la réalisation de ses affiches, Szymon engrange toutes ses découvertes inédites liées au design, le Best of Wikipedia est actualisé deux fois par jour par son auteur qui rassemble ses « meilleures lectures ». Les exemples sont légions.

Sous un angle plus technique, le système autorisant la republication de fils RSS, un faisceau d'informations thématiques peut être rassemblé sous la même bannière et constituer un flux de nouvelles spécialisées, comme les seriousgames, les technologies liées au web3, etc. L'activisme politique n'est pas en reste puisque peuvent être republiés automatiquement des fils Twitter (ex. : Recriweb) dont on a vu récemment avec les événements d'Iran de juin 2009 qu'ils pouvaient traverser les frontières et être communiqués très rapidement à des millions de connectés.

\section{De l'auteur institutionnalisé à l'auteur incarné}

L'absence d'encadrement institutionnel éditorial qui caractérise la condition première des blogs a deux conséquences en sus de la promotion des écritures ordinaires et éphémères : l'exhibition d'un processus autoritatif et l'incarnation de l'auteur dans les textes.

On reconnaît une construction auctoriale classique qui se produit selon deux mouvements distingués ici mais se déroulant en réalité de manière enchevêtrée. Il y a d'abord l'établissement d'une pratique par une production régulière de billets. On peut dire que c'est l'écrivain en tant que scripteur qui va installer l'auteur. Celui-ci va progressivement s'affirmer et, s'il ne l'a pas fait dès la phase introductive, se dévoiler en apportant des détails sur ses intérêts, avec des indications de lieux et d'événements auxquels il a participé. 
Puis, on peut affirmer que l'auteur construit l'écrivain lorsque les affirmations à la première personne de type « je » ou « moi » ou des interrogations comme « savez-vous que » commencent à être maîtrisées et entrent dans une stratégie d'écriture (comme Graphic qui s'adresse d'abord à un public constitué par les étudiants de sa promo, voir fig. 3 et 4). Ces entités linguistiques sont le signe d'une installation d'auteur au sein de son œuvre, qui est aussi repérable dans les blogs anonymes par les métalepses narratives; ainsi Robomixer :

Je m'invente un personnage fictif issu de choses improbables : « robomixer »! Pas un super héros, ni une triste épave. On a trop tendance à voir les choses en noir - comme si le monde allait s'écrouler - et il y a pourtant de quoi. Et l'on a aussi de formidables raisons de rire - au moins de désespoir.

Enfin, il faut signaler que les blogueurs « réussis », c'est-à-dire identifiés en tant qu'amateurs éclairés ou spécialistes d'un domaine-comme RachelHills qui tient à jour le Best Australian feminist blog - laissent de multiples traces sur le Web. Cela peut résulter d'une stratégie d'identification ou bien être la résultante d'une activité intense d'écriture comme Butterflyeffects qui maintient plus d'une vingtaine de blogs de manière anonyme.

Ainsi aux côtés de l'auteur porté par l'éditeur, reconnu par les institutions culturelles, un nouveau profil commence à s'imposer : celui de l'auteur incarné dont la notoriété se mesure à l'amplitude de la conversation provoquée par ses billets, mesurable par les re-blogs, les citations, les « on aime », les « trackbacks », jusqu'à ce qu'il soit répertorié par les médias traditionnels (journaux, radios, télévision) et intégré dans la chaîne de l'autorité. Cet auteur disséminateur bâtit une œuvre-flux plutôt qu'un patrimoine, à partir d'objets remaniés, remixés, recomposés.

En guise de conclusion nous retiendrons que le conditionnement de l'expression par l'outil est tel qu'il parvient à formater le genre. Le blogging provoque des métalepses d'auteur en prescrivant des comportements d'autoréférence. Les monographies des blogueurs de Tumblr sont le résultat de consignes d'écriture implicites qui encouragent les collections sous forme de listes par des opérations de re-publication. La fonction éditoriale disparaît au profit du logiciel de publication en ligne qui donne les formes d'un nouveau genre documentaire.

Évelyne Broudoux Université de Versailles-Saint-Quentin-DICEN 